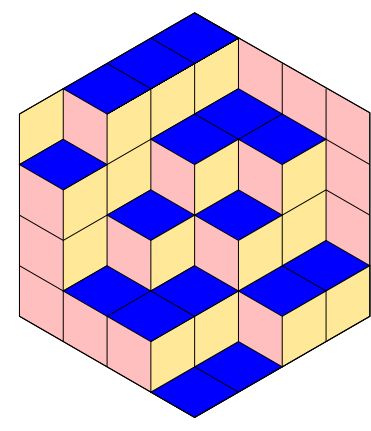

ALGEBRAIC COMBINATORICS

Isabella Novik \& Hailun Zheng

The stresses on centrally symmetric complexes and the lower bound theorems

Volume 4, issue 3 (2021), p. 541-549.

<http://alco.centre-mersenne.org/item/ALCO_2021__4_3_541_0>

(C) The journal and the authors, 2021.

Some rights reserved.

(c) BY This article is licensed under the

Creative Commons ATtribution 4.0 International LiCEnSE.

http://creativecommons.org/licenses/by/4.0/

Access to articles published by the journal Algebraic Combinatorics on the website http://alco.centre-mersenne.org/ implies agreement with the Terms of Use (http://alco.centre-mersenne.org/legal/).

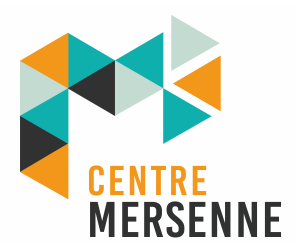

Algebraic Combinatorics is member of the Centre Mersenne for Open Scientific Publishing www.centre-mersenne.org 


\title{
The stresses on centrally symmetric complexes and the lower bound theorems
}

\author{
Isabella Novik \& Hailun Zheng
}

\begin{abstract}
In 1987, Stanley conjectured that if a centrally symmetric Cohen-Macaulay simplicial complex $\Delta$ of dimension $d-1$ satisfies $h_{i}(\Delta)=\left(\begin{array}{l}d \\ i\end{array}\right)$ for some $i \geqslant 1$, then $h_{j}(\Delta)=\left(\begin{array}{l}d \\ j\end{array}\right)$ for all $j \geqslant i$. Much more recently, Klee, Nevo, Novik, and Zheng conjectured that if a centrally symmetric simplicial polytope $P$ of dimension $d$ satisfies $g_{i}(\partial P)=\left(\begin{array}{c}d \\ i\end{array}\right)-\left(\begin{array}{c}d \\ i-1\end{array}\right)$ for some $d / 2 \geqslant i \geqslant 1$, then $g_{j}(\partial P)=\left(\begin{array}{l}d \\ j\end{array}\right)-\left(\begin{array}{c}d \\ j-1\end{array}\right)$ for all $d / 2 \geqslant j \geqslant i$. This note uses stress spaces to prove both of these conjectures.
\end{abstract}

\section{INTRODUCTION}

This paper is devoted to analyzing the cases of equality in Stanley's lower bound theorems on the face numbers of centrally symmetric Cohen-Macaulay complexes and centrally symmetric polytopes. All complexes considered in this paper are simplicial.

In the seventies, Stanley and Hochster (independently from each other) introduced the notion of Stanley-Reisner rings and started developing their theory, see $[5,8$, $9,10]$. In the fifty years since, this theory has become a major tool in the study of face numbers of simplicial complexes that resulted in a myriad of theorems and applications. Among them are a complete characterization of face numbers of CohenMacaulay (CM, for short) simplicial complexes [10], a complete characterization of flag face numbers of balanced CM complexes [3, 11], and a complete characterization of face numbers of simplicial polytopes $[2,12]$, to name just a few.

A simplicial complex $\Delta$ is called centrally symmetric (or cs) if its vertex set $V$ is endowed with a free involution $\alpha: V \rightarrow V$ that induces a free involution on the set of all non-empty faces of $\Delta$. Motivated by the desire to understand face numbers of cs simplicial polytopes as well as to find a complete characterization of face numbers of cs CM complexes, Stanley [13, Theorems 3.1 and 4.1] proved the following Lower Bound Theorem:

Theorem 1.1. Let $\Delta$ be a $(d-1)$-dimensional cs CM simplicial complex. Then $h_{i}(\Delta) \geqslant\left(\begin{array}{c}d \\ i\end{array}\right)$ for all $1 \leqslant i \leqslant d$. Furthermore, if $\Delta$ is the boundary complex of a $d$-dimensional cs simplicial polytope, then $g_{i}(\Delta) \geqslant\left(\begin{array}{l}d \\ i\end{array}\right)-\left(\begin{array}{c}d \\ i-1\end{array}\right)$ for all $1 \leqslant i \leqslant d / 2$.

\footnotetext{
Manuscript received 28th August 2020, revised 10th January 2021, accepted 11th January 2021.
}

KEywords. Cohen-Macaulay complexes, polytopes, centrally symmetric, face numbers, stress spaces.

ACKNOWLEDGEMENTS. Novik's research is partially supported by NSF grants DMS-1664865 and DMS-1953815, and by Robert R. \& Elaine F. Phelps Professorship in Mathematics.

Zheng's research is partially supported by a postdoctoral fellowship from ERC grant 716424-CASe. 
These inequalities are sharp: indeed, the boundary complex of the $d$-cross-polytope has $h_{i}=\left(\begin{array}{c}d \\ i\end{array}\right)$ for all $i$ and $g_{i}=\left(\begin{array}{c}d \\ i\end{array}\right)-\left(\begin{array}{c}d \\ i-1\end{array}\right)$ for all $1 \leqslant i \leqslant d / 2$. Stanley also proposed the following conjecture [13, Conjecture 3.5], which he verified in the case that $j$ is even or $j-i$ is even:

ConjeCture 1.2. Let $\Delta$ be a $(d-1)$-dimensional cs CM simplicial complex. Suppose $h_{i}(\Delta)=\left(\begin{array}{l}d \\ i\end{array}\right)$ for some $i \geqslant 1$. Then $h_{j}(\Delta)=\left(\begin{array}{l}d \\ j\end{array}\right)$ for all $j \geqslant i$.

Much more recently, Klee, Nevo, Novik, and Zheng [6, Conjecture 8.5] posited a conjecture that is similar in spirit, which they verified for $i=2$ (the case of $i=1$ is very easy):

Conjecture 1.3. Let $\Delta$ be the boundary complex of a d-dimensional cs simplicial polytope. Suppose $g_{i}(\Delta)=\left(\begin{array}{c}d \\ i\end{array}\right)-\left(\begin{array}{c}d \\ i-1\end{array}\right)$ for some $d / 2 \geqslant i \geqslant 1$. Then $g_{j}(\Delta)=\left(\begin{array}{c}d \\ j\end{array}\right)-\left(\begin{array}{c}d \\ j-1\end{array}\right)$ for all $d / 2 \geqslant j \geqslant i$.

In this note we prove both conjectures in full generality. The proofs are given in Section 3. Along the way, we show that any complex $\Delta$ satisfying conditions of Conjecture 1.2 contains the boundary complex of a $d$-cross-polytope as a subcomplex - a fact that might be of independent interest. Our proof utilizes the theory of stress spaces developed by Lee [7]. Specifically, the $h$-numbers of a Cohen-Macaulay complex $\Delta$ can be viewed as the dimensions of certain spaces of linear stresses on $\Delta$ while the $g$-numbers of the boundary complex of a simplicial polytope are the dimensions of spaces of affine stresses. A key observation is that if $\Delta$ is a $(d-1)$-dimensional $c s$ CM complex, then $h_{i}(\Delta)=\left(\begin{array}{l}d \\ i\end{array}\right)$ if and only if all linear $i$-stresses on $\Delta$ are symmetric; similarly, if $\Delta$ is the boundary complex of a $d$-dimensional $c s$ simplicial polytope, then $g_{i}(\Delta)=\left(\begin{array}{c}d \\ i\end{array}\right)-\left(\begin{array}{c}d \\ i-1\end{array}\right)$ if and only if all affine $i$-stresses on $\Delta$ are symmetric, see the discussion in Section 2. Both conjectures then follow from the main result of the paper asserting that for an arbitrary cs simplicial complex $\Delta$, if $\Theta$ is a set of linear forms satisfying certain conditions and if for some $i>1$, all $i$-stresses on $\Delta$ computed w.r.t. $\Theta$ are symmetric, then so are all $j$-stresses on $\Delta$ for any $j \geqslant i$, see Theorem 3.5.

\section{Setting the Stage}

We review several definitions and results on simplicial complexes, Stanley-Reisner rings, stress spaces, and Cohen-Macaulayness, as well as prepare ground for the proofs. For all undefined terminology we refer the reader to $[7,15]$.

$\mathrm{A}$ (n abstract) simplicial complex $\Delta$ on the ground set $V$ is a collection of subsets of $V$ that is closed under inclusion; $v$ is a vertex of $\Delta$ if $\{v\} \in \Delta$, but not all elements of $V$ are required to be vertices. The elements of $\Delta$ are called faces. The dimension of a face $\tau \in \Delta$ is $\operatorname{dim} \tau:=|\tau|-1$. The dimension of $\Delta, \operatorname{dim} \Delta$, is the maximum dimension of its faces. A face of a simplicial complex $\Delta$ is a facet if it is maximal w.r.t. inclusion. We say that $\Delta$ is pure if all facets of $\Delta$ have the same dimension. To simplify notation, for a face that is a vertex, we write $v$ instead of $\{v\}$; we also define the following two subcomplexes of $\Delta$ called the star of $v$ and the link of $v$ in $\Delta$ : $\operatorname{st}_{\Delta}(v)=\operatorname{st}(v):=\{\sigma \in \Delta: \sigma \cup v \in \Delta\}$ and $\operatorname{lk}_{\Delta}(v)=\operatorname{lk}(v):=\left\{\sigma \in \mathrm{st}_{\Delta}(v): v \notin \sigma\right\}$.

Let $\Delta$ be a $(d-1)$-dimensional simplicial complex. For $-1 \leqslant i \leqslant d-1$, the $i$ th $f$-number of $\Delta, f_{i}=f_{i}(\Delta)$, denotes the number of $i$-dimensional faces of $\Delta$. The $h$-numbers of $\Delta, h_{i}=h_{i}(\Delta)$ for $0 \leqslant i \leqslant d$, are defined by the relation $\sum_{i=0}^{d} h_{i} \lambda^{d-i}=$ $\sum_{i=0}^{d} f_{i-1}(\lambda-1)^{d-i}$. Finally, the $g$-numbers of $\Delta$ are $g_{0}(\Delta):=1$ and $g_{i}(\Delta):=h_{i}(\Delta)-$ $h_{i-1}(\Delta)$ for $1 \leqslant i \leqslant d / 2$.

Let $\Delta$ be a simplicial complex on the ground set $V$. Let $X=\left\{x_{v}: v \in V\right\}$ be the set of variables and let $\mathbb{R}[X]$ be the polynomial ring over the real numbers $\mathbb{R}$ in 
variables $X$. The Stanley-Reisner ideal of $\Delta$ is defined as

$$
I_{\Delta}=\left(x_{v_{1}} x_{v_{2}} \ldots x_{v_{i}}:\left\{v_{1}, v_{2}, \ldots, v_{i}\right\} \notin \Delta\right),
$$

i.e. it is the ideal generated by the squarefree monomials corresponding to non-faces of $\Delta$. The Stanley-Reisner ring of $\Delta$ is $\mathbb{R}[\Delta]:=\mathbb{R}[X] / I_{\Delta}$. The ring $\mathbb{R}[\Delta]$ has an $\mathbb{N}$ grading: $\mathbb{R}[\Delta]=\bigoplus_{i=0}^{\infty} \mathbb{R}[\Delta]_{i}$, where the $i$ th graded component $\mathbb{R}[\Delta]_{i}$ is the space of homogeneous elements of degree $i$ in $\mathbb{R}[\Delta]$. In general, for an $\mathbb{N}$-graded vector space $M$, denote by $M_{i}$ the $i$ th graded component of $M$.

Let $\Delta$ be a simplicial complex and let $\Theta=\theta_{1}, \ldots, \theta_{\ell}$ be a sequence of linear forms in $\mathbb{R}[X]$, where $\ell$ is a nonnegative integer. Denote the quotient $\mathbb{R}[\Delta] / \Theta \mathbb{R}[\Delta]$ by $\mathbb{R}(\Delta, \Theta)$.

For our proofs, we will work in the dual setting of stress spaces developed by Lee [7], see also [1, Section 3]. It should also be mentioned that stress spaces are essentially the same objects as inverse systems in commutative algebra - the notion that goes back to Macaulay; see [4, Theorem 21.6 and Exercise 21.7]. Observe that a variable $x_{v}$ acts on $\mathbb{R}[X]$ by $\frac{\partial}{\partial x_{v}}$; for brevity, we will denote this operator by $\partial_{x_{v}}$. More generally, if $c(X)=\sum_{v \in V} c_{v} x_{v}$ is a linear form in $\mathbb{R}[X]$, then we define

$$
\begin{aligned}
\partial_{c(X)}: \mathbb{R}[X] & \rightarrow \mathbb{R}[X], \\
w & \mapsto \sum_{v \in V} c_{v} \cdot \partial_{x_{v}} w=\sum_{v \in V} c_{v} \frac{\partial w}{\partial x_{v}} .
\end{aligned}
$$

For a monomial $\mu \in \mathbb{R}[X]$, the support of $\mu$ is $\operatorname{supp}(\mu)=\left\{v \in V: x_{v} \mid \mu\right\}$. A homogeneous polynomial $w \in \mathbb{R}[X]$ of degree $i$ is called an $i$-stress on $\Delta$ w.r.t. $\Theta=$ $\theta_{1}, \ldots, \theta_{\ell}$ if it satisfies the following conditions:

- Every term $\mu$ of $w$ is supported on a face of $\Delta: \operatorname{supp}(\mu) \in \Delta$, and

- $\partial_{\theta_{k}} w=0$ for all $k=1, \ldots, \ell$.

The support of an $i$-stress $w, \operatorname{supp}(w)$, is the subcomplex of $\Delta$ generated by the support of all terms of $w$. We say that a face $F \in \Delta$ participates in a stress $w$ if $F \in \operatorname{supp}(w)$. We also say that a stress $w$ lives on a subcomplex $\Gamma$ of $\Delta$ if $\operatorname{supp}(w) \subseteq \Gamma$.

Denote the set of all $i$-stresses on $\Delta$ w.r.t. $\Theta$ by $\mathcal{S}(\Delta, \Theta)_{i}$. This set is a vector space $[1,7]$; it is a subspace of $\mathbb{R}[X]$. In fact, $\mathcal{S}(\Delta, \Theta)_{i}$ is the orthogonal complement of $\left(I_{\Delta}+(\Theta)\right)_{i}$ in $\mathbb{R}[X]_{i}$ w.r.t. a certain inner product on $\mathbb{R}[X]_{i}$, see $[7$, Section 3]. Thus, as a vector space, $\mathcal{S}(\Delta, \Theta)_{i}$ is canonically isomorphic to $\mathbb{R}(\Delta, \Theta)_{i}$. (For an alternative approach using the Weil duality, see [1, Section 3].) Another very useful and easy fact is that for every linear form $c(X) \in \mathbb{R}[X]$, the operator $\partial_{c(X)}$ maps $\mathcal{S}(\Delta, \Theta)_{i}$ into $\mathcal{S}(\Delta, \Theta)_{i-1}$, that is, if $w$ is a stress, then so is $\partial_{c(X)} w$. This follows from the fact that $\partial_{\theta_{k}}$ and $\partial_{c(X)}$ commute, and that a subset of a face of $\Delta$ is a face of $\Delta$.

Stresses are convenient to work with for the following reason: if $\Gamma$ is a subcomplex of $\Delta$ (considered as a complex on the same ground set $V$ as $\Delta$ ), then there is a natural surjective homomorphism $\rho: \mathbb{R}[\Delta] \rightarrow \mathbb{R}[\Gamma]$; it induces a surjective homomorphism $\mathbb{R}(\Delta, \Theta) \rightarrow \mathbb{R}(\Gamma, \Theta)$. On the level of stress spaces, the situation is much easier to describe: $\mathcal{S}(\Gamma, \Theta)_{i}$ is a subspace of $\mathcal{S}(\Delta, \Theta)_{i}$.

A simplicial complex $\Delta$ is centrally symmetric or $c s$ if its ground set is endowed with a free involution $\alpha: V \rightarrow V$ that induces a free involution on the set of all nonempty faces of $\Delta$. In more detail, for all non-empty faces $\tau \in \Delta$, the following holds: $\alpha(\tau) \in \Delta, \alpha(\tau) \neq \tau$, and $\alpha(\alpha(\tau))=\tau$. To simplify notation, we write $\alpha(\tau)=-\tau$ and refer to $\tau$ and $-\tau$ as antipodal faces of $\Delta$.

A large family of cs simplicial complexes is given by cs simplicial polytopes. A polytope $P \subset \mathbb{R}^{d}$ is the convex hull of a set of finitely many points in $\mathbb{R}^{d}$. We will always assume that $P$ is $d$-dimensional. A proper face of $P$ is the intersection of $P$ with a supporting hyperplane. A polytope $P$ is called simplicial if all of its proper faces 
are geometric simplices, i.e. convex hulls of affinely independent points. We identify each face of a simplicial polytope $P$ with the set of its vertices. The boundary complex of $P$, denoted $\partial P$, is then the simplicial complex consisting of the empty set along with the vertex sets of proper faces of $P$. A polytope $P$ is called $c s$ if $P=-P$; in this case, the complex $\partial P$ is a cs simplicial complex w.r.t. the natural involution. An important example is $\partial \mathcal{C}_{d}^{*}$ - the boundary complex of a $d$-cross-polytope $\mathcal{C}_{d}^{*}:=$ $\operatorname{conv}\left( \pm p_{1}, \pm p_{2}, \ldots, \pm p_{d}\right)$, where $p_{1}, \ldots, p_{d}$ are affinely independent points in $\mathbb{R}^{d} \backslash\{0\}$. As an abstract simplicial complex, $\partial \mathcal{C}_{d}^{*}$ is the $d$-fold suspension of $\{\varnothing\}$. It is easy to check that $h_{j}\left(\partial \mathcal{C}_{d}^{*}\right)=\left(\begin{array}{l}d \\ j\end{array}\right)$ for all $0 \leqslant j \leqslant d$, and so $g_{j}\left(\partial \mathcal{C}_{d}^{*}\right)=\left(\begin{array}{c}d \\ j\end{array}\right)-\left(\begin{array}{c}d \\ j-1\end{array}\right)$ for all $1 \leqslant j \leqslant d / 2$.

The free involution $\alpha$ on a cs complex $\Delta$ induces the free involution on $X$ via $\alpha\left(x_{v}\right)=x_{-v}$, which in turn induces a $\mathbb{Z} / 2 \mathbb{Z}$-action on $\mathbb{R}[X]$ and $\mathbb{R}[\Delta]$. For any $\mathbb{R}$ vector space $W$ endowed with such an action $\alpha$, one has $W=W^{+} \oplus W^{-}$, where $W^{+}:=\{w \in W: w=\alpha(w)\}$ and $W^{-}:=\{w \in W: w=-\alpha(w)\}$. Thus, $\mathbb{R}[\Delta]_{i}=$ $\mathbb{R}[\Delta]_{i}^{+} \oplus \mathbb{R}[\Delta]_{i}^{-}$. As $\mathbb{R}[\Delta]_{i}^{+} \cdot \mathbb{R}[\Delta]_{j}^{-} \subseteq \mathbb{R}[\Delta]_{i+j}^{-}$, and similar inclusions hold for all choices of plus and minus signs, it follows that $\mathbb{R}[\Delta]$ has an $(\mathbb{N} \times \mathbb{Z} / 2 \mathbb{Z})$-grading.

Let $\Delta$ be a cs simplicial complex with an involution $\alpha$, and let $\Theta=\theta_{1}, \ldots, \theta_{\ell}$ consist of linear forms that are homogeneous w.r.t. the $(\mathbb{N} \times \mathbb{Z} / 2 \mathbb{Z})$-grading. Since $\alpha\left(I_{\Delta}+(\Theta)\right)=I_{\Delta}+(\Theta)$ and since for any $w, w^{\prime} \in \mathbb{R}[X]_{i},\left\langle\alpha(w), \alpha\left(w^{\prime}\right)\right\rangle=\left\langle w, w^{\prime}\right\rangle$, where $\langle-,-\rangle$ is the inner product from [7, Section 3] used to define the isomorphism $\Phi_{i}$ between $\mathbb{R}(\Delta, \Theta)_{i}$ and $\mathcal{S}(\Delta, \Theta)_{i}$, it follows that $\alpha$ also acts on $\mathcal{S}(\Delta, \Theta)_{i}$ and that this action commutes with $\Phi_{i}$. Hence, $\mathcal{S}(\Delta, \Theta)_{i}=\mathcal{S}(\Delta, \Theta)_{i}^{+} \oplus \mathcal{S}(\Delta, \Theta)_{i}^{-}$, where the subspaces $\mathcal{S}(\Delta, \Theta)_{i}^{+}$and $\mathcal{S}(\Delta, \Theta)_{i}^{-}$of $\mathcal{S}(\Delta, \Theta)_{i}$ are isomorphic (as vector spaces) to $\mathbb{R}(\Delta, \Theta)_{i}^{+}$and $\mathbb{R}(\Delta, \Theta)_{i}^{-}$, resp. We refer to the elements of $\mathcal{S}(\Delta, \Theta)_{i}^{+}$as symmetric $i$-stresses.

For certain classes of simplicial complexes and a certain choice of $\Theta$, the dimensions of stress spaces are well understood. This requires a few additional definitions. Let $\Delta$ be a $(d-1)$-dimensional simplicial complex. A sequence $\Theta=\theta_{1}, \ldots, \theta_{\ell}$ of linear forms in $\mathbb{R}[X]$ is called a linear system of parameters of $\Delta$ (or l.s.o.p., for short) if $\ell=d$ and $\mathbb{R}(\Delta, \Theta)$ is a finite-dimensional $\mathbb{R}$-vector space. We say that $\Delta$ is Cohen-Macaulay (or CM, for short) if for some (equivalently, every) l.s.o.p. $\Theta=\theta_{1}, \theta_{2}, \ldots, \theta_{d}$ of $\Delta$,

$$
\operatorname{dim}_{\mathbb{R}} \mathbb{R}(\Delta, \Theta)_{i}=h_{i}(\Delta), \quad \forall 0 \leqslant i \leqslant d .
$$

In particular, if $\Delta$ is $\mathrm{CM}$ and $\Theta$ is an l.s.o.p. of $\Delta$, then $\mathcal{S}(\Delta, \Theta)_{i}$ has dimension $h_{i}(\Delta)$. Following [7], when $\Theta$ is an l.s.o.p. of $\Delta$, we will refer to elements of $\mathcal{S}(\Delta, \Theta)_{i}$ as linear $i$-stresses.

It is worth mentioning that there are other equivalent definitions of CM complexes. The most standard one is that $\Delta$ is CM if some (equivalently, every) l.s.o.p. of $\Delta$ is a regular sequence for the $\mathbb{R}[X]$-module $\mathbb{R}[\Delta]$. It is also worth mentioning that CM complexes have a topological characterization due to Reisner [8]. This characterization implies, for instance, that CM complexes are pure, that stars and links of CM complexes are also CM, and that the boundary complexes of simplicial polytopes are CM. ${ }^{(1)}$

Stanley [13] showed that if $\Delta$ is a cs simplicial complex, then there exists an l.s.o.p. $\Theta=\theta_{1}, \ldots, \theta_{d}$ of $\Delta$ with the property that each $\theta_{k}$ lies in $\mathbb{R}[X]_{1}^{-}$. We refer to such $\Theta$ as Stanley's special l.s.o.p. of $\Delta$; this object plays a crucial role in the proof of Conjecture 1.2. In the case that $\Delta=\partial P$ is the boundary complex of a cs $d$-polytope

${ }^{(1)}$ For any field $\mathbf{k}$, one may analogously define the rings $\mathbf{k}[\Delta]$ and $\mathbf{k}(\Delta, \Theta)$ as well as the notion of $\Delta$ being CM over $\mathbf{k}$. However, it follows from Reisner's criterion along with the universal coefficient theorem that if $\Delta$ is CM over some field $\mathbf{k}$, then $\Delta$ is CM over $\mathbb{R}$, i.e. $\Delta$ satisfies the definition given above. In other words, no generality is lost by working over $\mathbb{R}$. 
$P \subset \mathbb{R}^{d}$, there is a canonical choice of Stanley's special l.s.o.p. $\theta_{1}, \ldots, \theta_{d}$ of $\Delta$ defined as follows: for $k=1, \ldots, d$,

(1) $\quad \theta_{k}=\sum_{v \in V} a_{v, k} x_{v}$, where $a_{v, k}$ is the $k$-th coordinate of vertex $v \in P \subset \mathbb{R}^{d}$.

To prove Conjecture 1.3 we will consider stresses on $\partial P$ w.r.t. $\widetilde{\Theta}=\theta_{1}, \ldots, \theta_{d}, \theta_{d+1}$, where $\theta_{1}, \ldots, \theta_{d}$ are defined by (1) and $\theta_{d+1}:=\sum_{v \in V} x_{v}$ is an element of $\mathbb{R}[X]_{1}^{+}$. We will refer to $\widetilde{\Theta}$ as the set of canonical linear forms associated with $P$. Following [7], the $i$-stresses on $\partial P$ w.r.t. $\widetilde{\Theta}$ are called affine $i$-stresses.

The two main results of [13] (see proofs of Theorems 3.1 and 4.1 there) are the following Lower Bound Theorems for cs CM complexes and cs simplicial polytopes.

TheOREM 2.1. Let $\Delta$ be a $(d-1)$-dimensional cs CM simplicial complex, and let $\Theta$ be Stanley's special l.s.o.p. of $\Delta$. Then

$$
\operatorname{dim}_{\mathbb{R}} \mathbb{R}(\Delta, \Theta)_{i}^{-}=\frac{1}{2}\left(h_{i}(\Delta)-\left(\begin{array}{c}
d \\
i
\end{array}\right)\right) \quad \text { for all } 1 \leqslant i \leqslant d .
$$

In particular, $h_{i}(\Delta) \geqslant\left(\begin{array}{l}d \\ i\end{array}\right)$ for all $1 \leqslant i \leqslant d$.

Furthermore, if $\Delta=\partial P$ for some cs simplicial polytope $P$ and $\widetilde{\Theta}$ is the set of canonical linear forms associated with $P$, then

$$
\operatorname{dim}_{\mathbb{R}} \mathbb{R}(\Delta, \widetilde{\Theta})_{i}^{-}=\frac{1}{2}\left(g_{i}(\Delta)-\left(\begin{array}{c}
d \\
i
\end{array}\right)+\left(\begin{array}{c}
d \\
i-1
\end{array}\right)\right) \quad \text { for all } 1 \leqslant i \leqslant d / 2
$$

In particular, $g_{i}(\Delta) \geqslant\left(\begin{array}{l}d \\ i\end{array}\right)-\left(\begin{array}{c}d \\ i-1\end{array}\right)$ for all $1 \leqslant i \leqslant d / 2$.

Using the language of stresses, Theorem 2.1 leads to the following:

Corollary 2.2. Let $\Delta$ be a $(d-1)$-dimensional cs CM simplicial complex, let $\Theta$ be Stanley's special l.s.o.p. of $\Delta$, and let $1 \leqslant i \leqslant d$ be an integer. Then $h_{i}(\Delta)=\left(\begin{array}{l}d \\ i\end{array}\right)$ if and only if all linear $i$-stresses on $\Delta$ are symmetric, i.e. $\mathcal{S}(\Delta, \Theta)_{i}=\mathcal{S}(\Delta, \Theta)_{i}^{+}$. Furthermore, if $\Delta=\partial P$ for some cs simplicial polytope $P, \widetilde{\Theta}$ is the set of canonical linear forms associated with $P$, and $1 \leqslant i \leqslant d / 2$, then $g_{i}(\Delta)=\left(\begin{array}{c}d \\ i\end{array}\right)-\left(\begin{array}{c}d \\ i-1\end{array}\right)$ if and only if all affine $i$-stresses on $\Delta$ are symmetric, i.e. $\mathcal{S}(\Delta, \widetilde{\Theta})_{i}=\mathcal{S}(\Delta, \widetilde{\Theta})_{i}^{+}$.

Proof. Recall that $\mathbb{R}(\Delta, \Theta)_{i}^{-} \cong \mathcal{S}(\Delta, \Theta)_{i}^{-}$and $\mathbb{R}(\partial P, \widetilde{\Theta})_{i}^{-} \cong \mathcal{S}(\partial P, \widetilde{\Theta})_{i}^{-}$. Theorem 2.1 then implies that $\mathcal{S}(\Delta, \Theta)_{i}^{-}=(0)$ if and only if $h_{i}(\Delta)=\left(\begin{array}{l}d \\ i\end{array}\right)$, and that $\mathcal{S}(\partial P, \widetilde{\Theta})_{i}^{-}=(0)$ if and only if $g_{i}(\Delta)=\left(\begin{array}{l}d \\ i\end{array}\right)-\left(\begin{array}{c}d \\ i-1\end{array}\right)$.

\section{Proof of the CONJeCtures}

With the tools of Section 2 at our disposal, we are ready to prove Conjectures 1.2 and 1.3. In fact, we prove a more general result, Theorem 3.5, from which the conjectures readily follow. To simplify notation, we assume that $V=\{ \pm 1, \pm 2, \ldots, \pm n\}$ and let $[j]$ denote the set $\{1,2, \ldots, j\}$. We also refer to the elements of $\mathbb{R}[X]_{i}^{+}$as symmetric i-polynomials.

We start with two simple lemmas.

Lemma 3.1. Let $\Delta$ be a cs simplicial complex and let $\Theta=\theta_{1}, \ldots, \theta_{\ell}$ be linear forms in $\mathbb{R}[X]$ that are homogeneous w.r.t. the $(\mathbb{N} \times \mathbb{Z} / 2 \mathbb{Z})$-grading. Let $v$ be a vertex of $\Delta$. If $w$ is a symmetric stress on $\Delta$ (w.r.t. $\Theta$ ) that lives on $\mathrm{st}(v)$, then, in fact, $w$ lives on $\operatorname{lk}(v) \cap \operatorname{lk}(-v)$. 
Proof. By the definition of cs complexes, $-v \notin \operatorname{st}(v)$. Thus the assumption that $w$ is symmetric and lives on $\operatorname{st}(v)$ implies that $w$ lives on $l \mathrm{k}(v)$. Now, since $w$ is symmetric, a face $F$ of $\Delta$ participates in $w$ if and only if $-F$ does. This together with the symmetry of $\Delta$ yields that $w$ lives on $\operatorname{lk}(v) \cap \operatorname{lk}(-v)$.

LEMMA 3.2. Let $\Delta$ be a cs simplicial complex, let $\Theta=\theta_{1}, \ldots, \theta_{\ell}$ be linear forms in $\mathbb{R}[X]$ that are homogeneous w.r.t. the $(\mathbb{N} \times \mathbb{Z} / 2 \mathbb{Z})$-grading, and let $w \in \mathcal{S}(\Delta, \Theta)_{i}$. If for every vertex $v, \partial_{x_{v}} w$ is a symmetric stress, then $w$ is a squarefree polynomial.

Proof. If $v$ is in the support of $w$, then $\partial_{x_{v}} w$ is a symmetric stress that lives on $\operatorname{st}(v)$. Hence by Lemma 3.1, $\partial_{x_{v}} w$ lives on $\operatorname{lk}(v)$. In particular, no term of $w$ is divisible by $x_{v}^{2}$.

The following two lemmas provide key ingredients for the proof of Theorem 3.5. For $k \in[n]$, we let $y_{k}$ denote $x_{k}+x_{-k}$.

LEMma 3.3. Let $w \in \mathbb{R}[X]_{i}$ be a squarefree symmetric polynomial such that $\partial_{x_{v}} w$ is symmetric for all vertices $v$. Then $w$ is a squarefree polynomial in $y_{1}, \ldots, y_{n}$, that is, $w$ can be written as

$$
w=\sum_{\substack{\tau \subseteq[n] \\|\tau|=i}} c_{\tau} \prod_{k \in \tau}\left(x_{k}+x_{-k}\right) \quad \text { for some } c_{\tau} \in \mathbb{R} .
$$

Proof. It is easy to prove by induction on $n$ that a squarefree polynomial $Q \in \mathbb{R}[X]$ is a polynomial in $y_{1}, \ldots, y_{n}$ if and only if $\partial_{x_{k}} Q=\partial_{x_{-k}} Q$ for all $k \in[n]$. Thus to prove the lemma, it is enough to check that our given $w$ satisfies $\partial_{x_{k}} w=\partial_{x_{-k}} w$ for all $k \in[n]$. Indeed, by symmetry of $w$ and $\partial_{x_{k}} w$, and by the definition of $\alpha$,

$$
\partial_{x_{k}} w=\alpha\left(\partial_{x_{k}} w\right)=\partial_{x_{-k}}(\alpha w)=\partial_{x_{-k}} w .
$$

The result follows.

LEMMA 3.4. Let $i \geqslant 1$ and let $w \in \mathbb{R}[X]_{i+1}$ be a squarefree polynomial such that for all vertices $v, \partial_{x_{v}} w$ is a polynomial in $y_{1}, \ldots, y_{n}$. Then $w$ is a squarefree polynomial in $y_{1}, \ldots, y_{n}$. In particular, $w$ is symmetric and can be expressed as

$$
w=\sum_{\substack{\sigma \subseteq[n] \\|\sigma|=i+1}} c_{\sigma} \prod_{k \in \sigma}\left(x_{k}+x_{-k}\right) \quad \text { for some } c_{\sigma} \in \mathbb{R} .
$$

Proof. By Lemma 3.3, the statement will follow if we show that $w$ is symmetric. To check this, write $w$ as $w=\sum c_{k_{1}, k_{2}, \ldots, k_{i+1}} x_{k_{1}} x_{k_{2}} \cdots x_{k_{i+1}}$ for some $c_{k_{1}, k_{2}, \ldots, k_{i+1}} \in \mathbb{R}$. The assumption that partial derivatives of $w$ are polynomials in $y_{1}, \ldots, y_{n}$ implies that $\partial_{x_{k_{2}}} \cdots \partial_{x_{k_{i+1}}} w$ is symmetric. Hence $c_{k_{1}, k_{2}, \ldots, k_{i+1}}=c_{-k_{1}, k_{2}, \ldots, k_{i+1}}$ (as they are coefficients of $x_{k_{1}}$ and $x_{-k_{1}}$ in $\left.\partial_{x_{k_{2}}} \cdots \partial_{x_{k_{i+1}}} w\right)$. Repeated applications of this argument imply that $c_{k_{1}, k_{2}, \ldots, k_{i+1}}=c_{-k_{1},-k_{2}, \ldots,-k_{i+1}}$. Thus, $w$ is symmetric.

We are now in a position to state and prove our main result.

THEOREM 3.5. Let $\Delta$ be a cs complex, and let $\Theta=\theta_{1}, \ldots, \theta_{\ell}$ be linear forms such that $\theta_{1}, \ldots, \theta_{\ell-1}$ are elements of $\mathbb{R}[X]_{1}^{-}$, and $\theta_{\ell}$ is either also in $\mathbb{R}[X]_{1}^{-}$or $\theta_{\ell}=\sum_{v \in V} x_{v}$. If for some integer $i>1$, all $i$-stresses on $\Delta$ w.r.t. $\Theta$ are symmetric, i.e. $\mathcal{S}(\Delta, \Theta)_{i}=$ $\mathcal{S}(\Delta, \Theta)_{i}^{+}$, then for all $j \geqslant i, \mathcal{S}(\Delta, \Theta)_{j}=\mathcal{S}(\Delta, \Theta)_{j}^{+}$. Furthermore, if $\mathcal{S}(\Delta, \Theta)_{j} \neq(0)$ for some $j>i$, then $\Delta$ contains the boundary complex of the $j$-cross-polytope as a subcomplex. 
Proof. It suffices to prove the statement for $j=i+1$. Let $w \in \mathcal{S}(\Delta, \Theta)_{i+1}$. For every vertex $v, \partial_{x_{v}} w \in \mathcal{S}(\Delta, \Theta)_{i}$, and so $\partial_{x_{v}} w$ is symmetric. Hence, by Lemma $3.2, w$ is squarefree.

Consider an edge $\left\{u_{1}, u_{2}\right\} \in \operatorname{supp}(w)$. Then $\partial_{x_{u_{1}}} w$ is a symmetric $i$-stress that lives on $\operatorname{st}\left(u_{1}\right)$, and so by Lemma 3.1 , it lives on $\operatorname{lk}\left(u_{1}\right) \cap \operatorname{lk}\left(-u_{1}\right)$. Consequently, the stress $\partial_{x_{u_{2}}} \partial_{x_{u_{1}}} w$ lives on $\operatorname{lk}\left(u_{1}\right) \cap \operatorname{lk}\left(-u_{1}\right)$. Since $\partial_{x_{u_{2}}} \partial_{x_{u_{1}}} w=\partial_{x_{u_{1}}} \partial_{x_{u_{2}}} w$, the same argument implies that it also lives on $\operatorname{lk}\left(u_{2}\right) \cap \operatorname{lk}\left(-u_{2}\right)$.

Let

$$
w^{\prime}:=\left(x_{u_{1}}+x_{-u_{1}}-x_{u_{2}}-x_{-u_{2}}\right) \cdot \partial_{x_{u_{2}}} \partial_{x_{u_{1}}} w .
$$

Our discussion shows that $\operatorname{supp}\left(w^{\prime}\right) \subseteq \Delta$. Furthermore, by our assumptions on $\Theta$ and the fact that $w \in \mathcal{S}(\Delta, \Theta)_{i+1}$, it follows that

$$
\partial_{\theta_{k}} w=0 \text { and } \partial_{\theta_{k}}\left(x_{u_{1}}+x_{-u_{1}}-x_{u_{2}}-x_{-u_{2}}\right)=0 \text { for all } 1 \leqslant k \leqslant \ell .
$$

Therefore, for all $1 \leqslant k \leqslant \ell$,

$$
\begin{aligned}
\partial_{\theta_{k}} w^{\prime}=\partial_{\theta_{k}}\left(x_{u_{1}}+x_{-u_{1}}-x_{u_{2}}\right. & \left.-x_{-u_{2}}\right) \cdot \partial_{x_{u_{2}}} \partial_{x_{u_{1}}} w \\
& +\left(x_{u_{1}}+x_{-u_{1}}-x_{u_{2}}-x_{-u_{2}}\right) \cdot \partial_{x_{u_{2}}} \partial_{x_{u_{1}}} \partial_{\theta_{k}} w=0 .
\end{aligned}
$$

Hence $w^{\prime} \in \mathcal{S}(\Delta, \Theta)_{i}$, and so it is symmetric. We conclude that

$$
\partial_{x_{u_{2}}} \partial_{x_{u_{1}}} w \in \mathcal{S}(\Delta, \Theta)_{i-1}^{+} \text {for any } u_{2} \in \operatorname{supp}\left(\partial_{x_{u_{1}}} w\right)
$$

Since the stress $\partial_{x_{u_{1}}} w$ itself is symmetric (indeed, it is an $i$-stress), Lemma 3.3 guarantees that $\partial_{x_{u_{1}}} w$ is of the form $\partial_{x_{u_{1}}} w=\sum_{\tau \subseteq[n],|\tau|=i} c_{\tau} \prod_{k \in \tau}\left(x_{k}+x_{-k}\right)$, for all $u_{1} \in \operatorname{supp}(w)$. It then follows from Lemma 3.4 that $w$ is a symmetric stress of the form $w=\sum_{\sigma \subseteq[n],|\sigma|=i+1} c_{\sigma} \prod_{k \in \sigma}\left(x_{k}+x_{-k}\right)$. In particular, we see from the definition of stresses that if $w \neq 0$, then the support of $w$ is the union of the boundary complexes of $(i+1)$-cross-polytopes. This completes the proof.

The proof of Conjectures 1.2 and 1.3 now readily follows. In the proof, we use linear and affine stresses, i.e. stresses w.r.t. Stanley's special l.s.o.p. $\Theta$ and w.r.t. the set of canonical linear forms $\widetilde{\Theta}$, respectively.

\section{THEOREM 3.6.}

(1) Let $d$ and $1 \leqslant i<d$ be integers. Let $\Delta$ be a cs CM complex of dimension $d-1$ with $h_{i}(\Delta)=\left(\begin{array}{l}d \\ i\end{array}\right)$. Then $h_{j}(\Delta)=\left(\begin{array}{l}d \\ j\end{array}\right)$ for all $i \leqslant j \leqslant d$.

(2) Let $d$ and $1 \leqslant i<d / 2$ be integers. If $\Delta=\partial P$ for some cs simplicial d-polytope $P$ and $g_{i}(\Delta)=\left(\begin{array}{c}d \\ i\end{array}\right)-\left(\begin{array}{c}d \\ i-1\end{array}\right)$, then $g_{j}(\Delta)=\left(\begin{array}{c}d \\ j\end{array}\right)-\left(\begin{array}{c}d \\ j-1\end{array}\right)$ for all $i \leqslant j \leqslant d / 2$.

Proof. We begin with the case of $i>1$. For the first part, let $\Theta$ be Stanley's special l.s.o.p. of $\Delta$. Since $h_{i}(\Delta)=\left(\begin{array}{c}d \\ i\end{array}\right)$, it follows from Corollary 2.2 that all linear $i$-stresses on $\Delta$ are symmetric. By Theorem 3.5 , all linear $j$-stresses (for any $j \geqslant i$ ) are also symmetric. Hence Corollary 2.2 yields the result. The proof of the second part is analogous: this time use $\widetilde{\Theta}$ - the set of canonical linear forms associated with $P$ and then apply Corollary 2.2 and Theorem 3.5 to affine stresses.

Next we deal with the case of $i=1$ in both parts. The assumption that $h_{1}(\Delta)=d$, or that $g_{1}(\Delta)=d-1$, is equivalent to $f_{0}(\Delta)=2 d$. Now, it follows easily from the definition of cs complexes that any cs complex on $2 d$ vertices is contained in the boundary complex of the $d$-cross-polytope, and so $\Delta \subseteq \partial \mathcal{C}_{d}^{*}$. Since $\Delta$ and $\partial \mathcal{C}_{d}^{*}$ are $\mathrm{CM}$ complexes of the same dimension, [14, Theorem 2.1] implies that $h_{j}(\Delta) \leqslant h_{j}\left(\partial \mathcal{C}_{d}^{*}\right)=$ $\left(\begin{array}{l}d \\ j\end{array}\right)$ for all $j$. On the other hand, according to Theorem $1.1, h_{j}(\Delta) \geqslant\left(\begin{array}{l}d \\ j\end{array}\right)$ for all $j$. Thus we must have $h_{j}(\Delta)=\left(\begin{array}{l}d \\ j\end{array}\right)$ for all $j$, and hence also $g_{j}(\Delta)=\left(\begin{array}{c}d \\ j\end{array}\right)-\left(\begin{array}{c}d \\ j-1\end{array}\right)$ for all $j$. (Moreover, that the two complexes $\Delta \subseteq \partial \mathcal{C}_{d}^{*}$ have the same $h$-numbers yields that they have the same $f$-numbers, and so, in fact, $\Delta \cong \partial \mathcal{C}_{d}^{*}$.) 
It is worth remarking that under the conditions of Theorem 3.6, we can say a bit more about $\Delta$ :

\section{COROLlary 3.7.}

(1) Let $\Delta$ be a $(d-1)$-dimensional cs CM simplicial complex with $h_{i}(\Delta)=\left(\begin{array}{l}d \\ i\end{array}\right)$ for some $1 \leqslant i<d$. Then $\Delta$ contains a subcomplex $\Gamma$ isomorphic to $\partial \mathcal{C}_{d}^{*}$. Furthermore, $\mathcal{S}(\Delta, \Theta)_{j}=\mathcal{S}(\Gamma, \Theta)_{j}$ for all $j \geqslant i$, where $\Theta$ is Stanley's special l.s.o.p. of $\Delta$.

(2) Let $\Delta=\partial P$ where $P$ is a cs simplicial d-polytope. If $g_{i}(\Delta)=\left(\begin{array}{c}d \\ i\end{array}\right)-\left(\begin{array}{c}d \\ i-1\end{array}\right)$ for some $1 \leqslant i \leqslant(d-2) / 2$, then $\Delta$ contains $\partial \mathcal{C}_{\lfloor d / 2\rfloor}^{*}$ as a subcomplex.

Proof. If $i=1$, then the proof of Theorem 3.6 implies that in both parts $\Delta \cong$ $\partial \mathcal{C}_{d}^{*}$. Thus assume that $i>1$. For the second statement, since by Theorem 3.6, $g_{\lfloor d / 2\rfloor}(\Delta)=\left(\begin{array}{c}d \\ \lfloor d / 2\rfloor\end{array}\right)-\left(\begin{array}{c}d \\ \lfloor d / 2\rfloor-1\end{array}\right)>0$, it follows that $\mathcal{S}(\Delta, \widetilde{\Theta})_{\lfloor d / 2\rfloor} \neq(0)$, where $\widetilde{\Theta}$ is the set of canonical linear forms associated with $P$. Since by our assumptions, $\mathcal{S}(\Delta, \widetilde{\Theta})_{i}=\mathcal{S}(\Delta, \widetilde{\Theta})_{i}^{+}$and $\lfloor d / 2\rfloor>i$, Theorem 3.5 guarantees that $\Delta$ contains $\partial \mathcal{C}_{\lfloor d / 2\rfloor}^{*}$ as a subcomplex.

The proof of the first statement is similar: since by Theorem 3.6, $h_{d}(\Delta)=1$, there is a non-zero linear $d$-stress $w$ on $\Delta$. Since $d>i$ and $\mathcal{S}(\Delta, \Theta)_{i}=\mathcal{S}(\Delta, \Theta)_{i}^{+}$, Theorem 3.5 implies that $\Delta$ must contain $\Gamma \cong \partial \mathcal{C}_{d}^{*}$ as a subcomplex. Then $\mathcal{S}(\Delta, \Theta)_{j} \supseteq \mathcal{S}(\Gamma, \Theta)_{j}$ for all $j$, and comparing the dimensions we see that, in fact, $\mathcal{S}(\Delta, \Theta)_{j}=\mathcal{S}(\Gamma, \Theta)_{j}$ for all $j \geqslant i$.

Acknowledgements. We are grateful to Satoshi Murai, Eran Nevo, Richard Stanley, and the two anonymous referees for comments on the previous versions of this note.

\section{REFERENCES}

[1] Karim Adiprasito, Combinatorial Lefschetz theorems beyond positivity, https://arxiv.org/ $\mathrm{abs} / 1812.10454$.

[2] Louis J. Billera and Carl W. Lee, A proof of the sufficiency of McMullen's conditions for $f$ vectors of simplicial convex polytopes, J. Combin. Theory Ser. A 31 (1981), no. 3, 237-255.

[3] Anders Björner, Peter Frankl, and Richard P. Stanley, The number of faces of balanced CohenMacaulay complexes and a generalized Macaulay theorem, Combinatorica 7 (1987), no. 1, 23-34.

[4] David Eisenbud, Commutative algebra. With a view toward algebraic geometry, Graduate Texts in Mathematics, vol. 150, Springer-Verlag, New York, 1995.

[5] Melvin Hochster, Cohen-Macaulay rings, combinatorics, and simplicial complexes, in Ring theory, II (Proc. Second Conf., Univ. Oklahoma, Norman, Okla., 1975), Lecture Notes in Pure and Appl. Math., vol. 26, Dekker, 1977, pp. 171-223.

[6] Steven Klee, Eran Nevo, Isabella Novik, and Hailun Zheng, A lower bound theorem for centrally symmetric simplicial polytopes, Discrete Comput. Geom. 61 (2019), no. 3, 541-561.

[7] Carl W. Lee, P.L.-spheres, convex polytopes, and stress, Discrete Comput. Geom. 15 (1996), no. $4,389-421$.

[8] Gerald A. Reisner, Cohen-Macaulay quotients of polynomial rings, Adv. Math. 21 (1976), no. 1, $30-49$.

[9] Richard P. Stanley, The upper bound conjecture and Cohen-Macaulay rings, Studies in Appl. Math. 54 (1975), no. 2, 135-142.

[10] Cohen-Macaulay complexes, Higher combinatorics (Proc. NATO Advanced Study Inst., Berlin, 1976), NATO Adv. Study Inst. Ser., Ser. C: Math. and Phys. Sci., vol. 31, 1977, pp. 5162 .

[11] , Balanced Cohen-Macaulay complexes, Trans. Amer. Math. Soc. 249 (1979), no. 1, $139-157$.

[12] $236-238$.

[13] , On the number of faces of centrally-symmetric simplicial polytopes, Graphs Combin. 3 (1987), no. 1, 55-66.

[14] A monotonicity property of $h$-vectors and $h^{*}$-vectors, European J. Combin. 14 (1993), no. $3,251-258$. 
The stresses on centrally symmetric complexes and the lower bound theorems

[15] _ Combinatorics and commutative algebra, second ed., Progress in Mathematics, vol. 41, Birkhäuser Boston, Inc., Boston, MA, 1996.

ISABELla NoviK, Department of Mathematics, University of Washington, Seattle, WA 981954350, USA

E-mail : novik@math.washington.edu

HAilun Zheng, Department of Mathematical Sciences, University of Copenhagen, Universitesparken 5, 2100 Copenhagen, Denmark

E-mail : hz@math.ku.dk 